\title{
SECOND HARMONICS AND COMPENSATION EFFECT IN CERAMIC SUPERCONDUCTORS
}

\author{
Mai Suan Li \\ Institute of Physics, Polish Academy of Sciences, Al. Lotnikow 32/46, 02-668 Warsaw, Poland
}

\begin{abstract}
A three-dimensional lattice of the Josephson junctions with a finite self-conductance is employed to model the ceramic superconductors. The nonlinear ac susceptibility and the compensation effect are studied by Monte Carlo simulations in this model. The compensation effect is shown to be due to the existence of the chiral glass phase. We demonstrate, in agreement with experiments, that this effect may be present in the ceramic superconductors which show the paramagnetic Meissner effect.
\end{abstract}

PACS numbers: 75.40.Gb, 74.72.-h

One of the most fascinating discoveries in condensed matter physics is the paramagnetic Meissner effect (PME) in certain ceramic superconductors [1,2]. The nature of the unusual paramagnetic behaviour may be related to the appearance of spontaneous suppercurrents (or of orbital moments) [3]. The latter appear due to the existence of $\pi$-junctions characterized by the negative Josephson couplings [3, 4. Furthermore, Sigrist and Rice argued that the PME in the high- $T_{c}$ superconductors is consistent with the $d$-wave superconductivity [5]. This effect is succesfully reproduced in a single loop model [5] as well as in a model of interacting junction-loops [6, ].

The mechanism of the PME based on the $d$-wave symmetry of the order parameter remains ambiguous because it is not clear why this effect could not be observed in many ceramic materials. More importantly, the paramagnetic response has been seen even in the conventional $\mathrm{Nb}[8-\sqrt{10}$ and $\mathrm{Al}$ [11] superconductors. In order to explain the PME in terms of conventional superconductivity one can employ the idea of the flux compression inside of a sample. Such phenomenon becomes possible in the presence of the inhomogeneities [12] or of the sample boundary [13]. Thus the intrinsic mechanism leading to the PME is still under debate [11,14].

Recently Heinzel et al. [15] have shown that the PME may be analyzed by the compensation technique based on the measurement of the second harmonics of the magnetic ac susceptibility. Their key observation is that the so called compensation effect (CE) appears only in the samples which show the PME but not in those which do not. Overall, this effect may be detected in the following way. The sample is cooled in the external dc field down to a low temperature and then the field is switched off. At the fixed low $T$ the second harmonics are monitored by applying the dc and ac fields to the sample. Due to the presence of non-zero spontaneous orbital moments the remanent magnetization or, equivalently, the internal field appears in the cooling process. If the direction of the external dc field is identical to that during the field cooled (FC) procedure, the induced shielding currents will reduce the remanence. Consequently, the absolute value of the second harmonics $\left|\chi_{2}\right|$ decreases until the signal of the second harmonics is minimized at a field $H_{d c}=H_{c o m}$. Thus the $\mathrm{CE}$ is a phenomenon in which the external and internal fields are compensated and the second harmonics become zero.

The goal of this paper is to explain the CE theoretically by Monte Carlo simulations. Our starting point is based on the possible existence of the chiral glass phase [16] in which the remanence necessary for observing the $\mathrm{CE}$ should occur in the cooling procedure. Such remanence phenomenon is similar to what happens in spin glass. Furthermore, the PME related to the CE can also be observed in the chiral glass phase [7, 16,17]. There are several experimental results 18 which appear to corroborate the existence of such a novel glassy phase in ceramic high- $T_{c}$ superconductors.

In the chiral glass phase the frustration due to existence of 0 - and $\pi$-junctions (0-junctions correspond to positive Josephson contact energies) leads to non-zero supercurrents [16]. The internal field (or the remanent magnetization) induced by the supercurrents in the cooling process from high temperatures to the chiral glass phase may compensate the external dc field.

We model ceramic superconductors by the threedimensional XY model of the Josephson network with finite self-inductance. We show that in the $\mathrm{FC}$ regime the $\mathrm{CE}$ appears in the samples which 
show the PME but not in those containing only 0junctions. In the zero field cooled (ZFC) regime decreasing the external dc field also gives rise to the $\mathrm{CE}$ in the frustrated ceramics. Both of these findings agree with the experimental data of Heinzel et al [15].

We neglect the charging effects of the grain and consider the following Hamiltonian [6,7]

$$
\begin{array}{r}
\mathcal{H}=-\sum_{<i j>} J_{i j} \cos \left(\theta_{i}-\theta_{j}-A_{i j}\right)+ \\
\frac{1}{2 \mathcal{L}} \sum_{p}\left(\Phi_{p}-\Phi_{p}^{e x t}\right)^{2}, \\
\Phi_{p}=\frac{\phi_{0}}{2 \pi} \sum_{<i j>}^{p} A_{i j}, A_{i j}=\frac{2 \pi}{\phi_{0}} \int_{i}^{j} \vec{A}(\vec{r}) d \vec{r}
\end{array}
$$

where $\theta_{i}$ is the phase of the condensate of the grain at the $i$-th site of a simple cubic lattice, $\vec{A}$ is the fluctuating gauge potential at each link of the lattice, $\phi_{0}$ denotes the flux quantum, $J_{i j}$ denotes the Josephson coupling between the $i$-th and $j$-th grains, $\mathcal{L}$ is the self-inductance of a loop (an elementary plaquette), while the mutual inductance between different loops is neglected. The first sum is taken over all nearestneighbor pairs and the second sum is taken over all elementary plaquettes on the lattice. Fluctuating variables to be summed over are the phase variables, $\theta_{i}$, at each site and the gauge variables, $A_{i j}$, at each link. $\Phi_{p}$ is the total magnetic flux threading through the $p$-th plaquette, whereas $\Phi_{p}^{e x t}$ is the flux due to an external magnetic field applied along the $z$-direction,

$$
\Phi_{p}^{e x t}= \begin{cases}H S & \text { if } p \text { is on the }<x y>\text { plane } \\ 0 & \text { otherwise }\end{cases}
$$

where $S$ denotes the area of an elementary plaquette. The external field $H$ includes the dc and ac parts and it is given by

$$
H=H_{d c}+H_{a c} \cos (\omega t) .
$$

It should be noted that the dc field is necessary to generate even harmonics.

In the present paper, we consider two models with two types of bond distributions. Model I: the sign of the Josephson couplings could be either positive (0junction) or negative ( $\pi$-junction) and the spin glass type bimodal $( \pm J)$ distribution of $J_{i j}$ is taken. The coexistence of 0 - and $\pi$-junctions gives rise to frustration even in zero external field and the chiral glass phase may occur at low temperatures [16]. Model II: the interactions $J_{i j}$ are assumed to be 'ferromagnetic' and distributed uniformly between 0 and $2 J$.
Obviously, there is no frustration in zero external field in this model. It has been also demonstrated that the PME is present in model I but not in model II [7].

The ac linear susceptibilty of models I and II has been studied [7] by Monte Carlo simulations. It was found that, due to the frustration, model I exhibits much stronger dissipation than model II in the low frequency regime. Here we go beyond our previous calculations of the linear ac susceptibility [7]. We study the dependence of the second harmonics as a function of the dc field. In this way, we can make a direct comparison with the CE observed in the experiments 15. The second harmonics of a similar Josephson network model with a finite selfinductance were considered by Wolf and Majhofer [19. However, these authors dealt with the twodimensional version of model II and the CE has not been studied. In this paper we are mainly interested in the $\mathrm{CE}$ in the frustrated three-dimensional system described by model I.

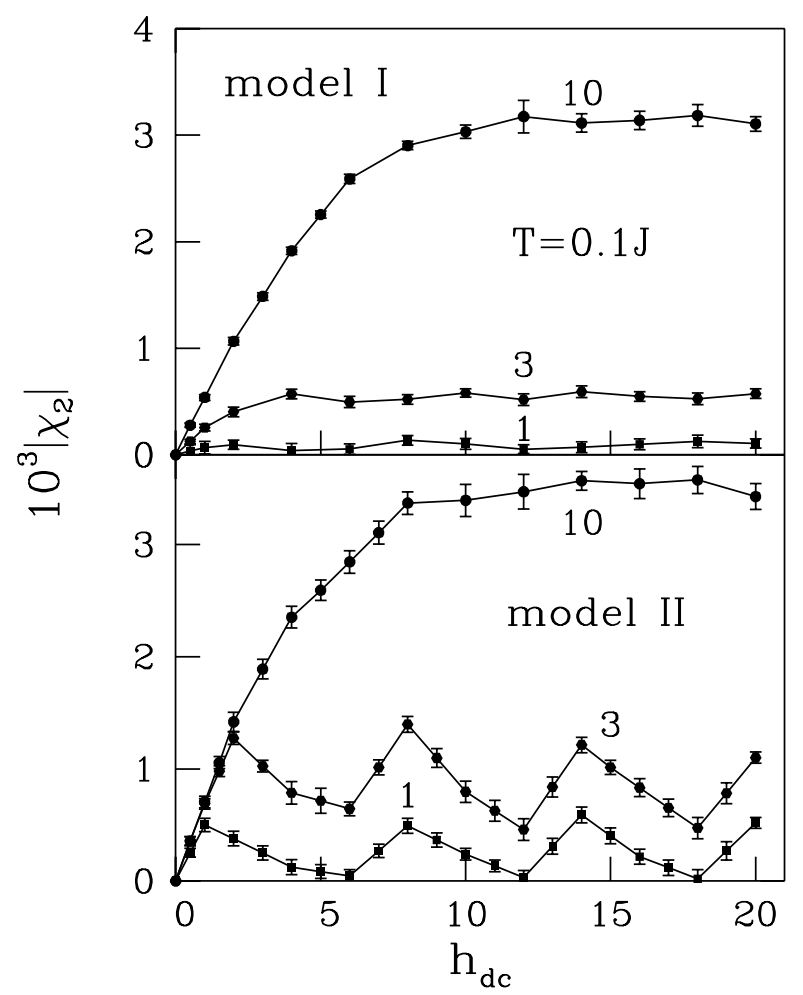

FIG. 1. The dependence of $\left|\chi_{2}\right|$ on $h_{d c}$ for model I and model II at $T=0.1$. The values of $\tilde{\mathcal{L}}$ are chosen to be equal to 1,3 and 10 as shown next to the curves. The results are averaged over 20 samples.

The dimensionless magnetization along the $z$-axis 
mormalized per plaquette, $\tilde{m}$, is given by

$$
\tilde{m}=\frac{1}{N_{p} \phi_{0}} \sum_{p<x y>}\left(\Phi_{p}-\Phi_{p}^{e x t}\right),
$$

where the sum is taken over all $N_{p}$ plaquettes on the $\langle x y\rangle$ plane of the lattice. The real and imaginary parts of the ac second order susceptibility $\chi_{2}^{\prime}(\omega)$ and $\chi_{2}^{\prime \prime}(\omega)$ are calculated as

$$
\begin{aligned}
& \chi_{2}^{\prime}(\omega)=\frac{1}{\pi h_{a c}} \int_{-\pi}^{\pi} \tilde{m}(t) \cos (2 \omega t) d(\omega t), \\
& \chi_{2}^{\prime \prime}(\omega)=\frac{1}{\pi h_{a c}} \int_{-\pi}^{\pi} \tilde{m}(t) \sin (2 \omega t) d(\omega t),
\end{aligned}
$$

where $t$ denotes the Monte Carlo time. The dimensionless ac field $h_{a c}$, dc field $h_{d c}$ and inductance $\tilde{\mathcal{L}}$ are defined as follows

$$
\begin{aligned}
h_{a c}=\frac{2 \pi H_{a c} S}{\phi_{0}}, h_{d c} & =\frac{2 \pi H_{d c} S}{\phi_{0}}, \\
\tilde{\mathcal{L}} & =\left(2 \pi / \phi_{0}\right)^{2} J \mathcal{L} .
\end{aligned}
$$

The dependence of $\tilde{\mathcal{L}}$ on the parameters of the system such as the critical current and the typical size of the grains is discussed in [7] 17.

Our results have been obtained by employing Monte Carlo simulations based on the standard Metropolis updating technique. While Monte Carlo simulations involve no real dynamics, one can still expect that they give useful information on the longtime behavior of the system. In fact, the amplitude of the ac field we use is much smaller than the typical energy of the dc part. On the other hand, the characteristic time for the sintered samples, which are believed to be captured by our model, is of order $10^{-12} s[19]$. This time has the same order of magnitude as a single Monte Carlo step. So the period of oscillations chosen in the present work is much longer than the characteristic time (see below). For such a weak and slowly changing ac field the system can be regarded as being in quasi-equilibrium and the Monte Carlo updating may be applied. A priori, the validity of this approximation is not clear but it may be justified by comparing our results with those obtained by other approaches to the dynamics such as considered in ref. 119. For the first harmonics, our method and the method of ref. [19] yield results that agree qualitatively. Furthermore, our results presented in Fig.1 for the second harmonics are also in a qualitative agreement with the corresponding results obtained by solving the equations of motion [19]. So one can expect that the standard Monte
Carlo may actually give reasonable results for the CE.

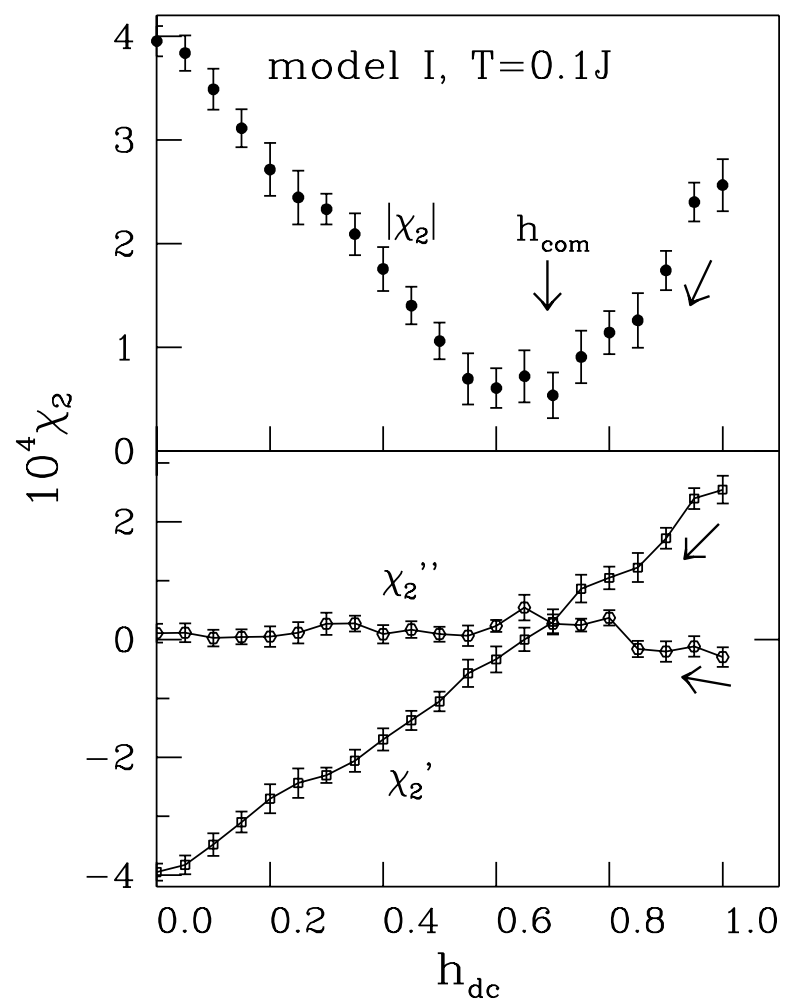

FIG. 2. The second harmonics of model I obtained after field cooling in a dc field $h_{d c}=1$ from $T=0.7$ to $T=0.1$. The temperature is reduced in steps of 0.05 . At the lowest $T=0.1$ the dc field used in cooling is switched off and the second harmonics are generated by applying the combined field (3). The $d c$ field is stepwise reduced from $h_{d} c=1$ to $h_{d} c=0$. The inductance is chosen to be equal to $\tilde{\mathcal{L}}=4$. The arrows indicate the sense of the changes in the dc field. The results are averaged over 40 samples and are qualitatively the same as those presented in Fig. 1 of Ref. [15].

We choose the gauge where the bond variables $A_{i j}$ along the $z$-direction are fixed to be zero. The lattice studied are simple cubic with $L \times L \times L$ sites and free boundary conditions are adopted. In all calculations presented below, we take $L=8$ and $\omega=0.001$. The sample average is taken over 20-40 independent bond realizations. $\chi_{2}(\omega)$ has been estimated following the procedure in [7,20]. Namely, at the beginnig of a given Monte Carlo run, we first switch on the field (3). Then, after waiting for initial $t_{0}$ Monte Carlo steps per spin (MCS), we start to monitor the time variation of the magnetization, $t_{0}$ is being chosen so that all transient phenomena can be considered 
extinct. We set $t_{0}$ to be $2 \times 10^{4}$ MCS. After passing the point $t=t_{0}, \tilde{m}(t)$ is averaged over typically 200 periods, each period contains $t_{T} \operatorname{MCS}\left(t_{T}=2 \pi / \omega\right)$. The real and imaginary parts of the second order ac susceptibility are then extracted via Eq. (5). We set $h_{a c}=0.1$, corresponding to $\approx 0.016$ flux quantum per plaquette. Smaller value of $h_{a c}$ turned out to leave the results almost unchanged.

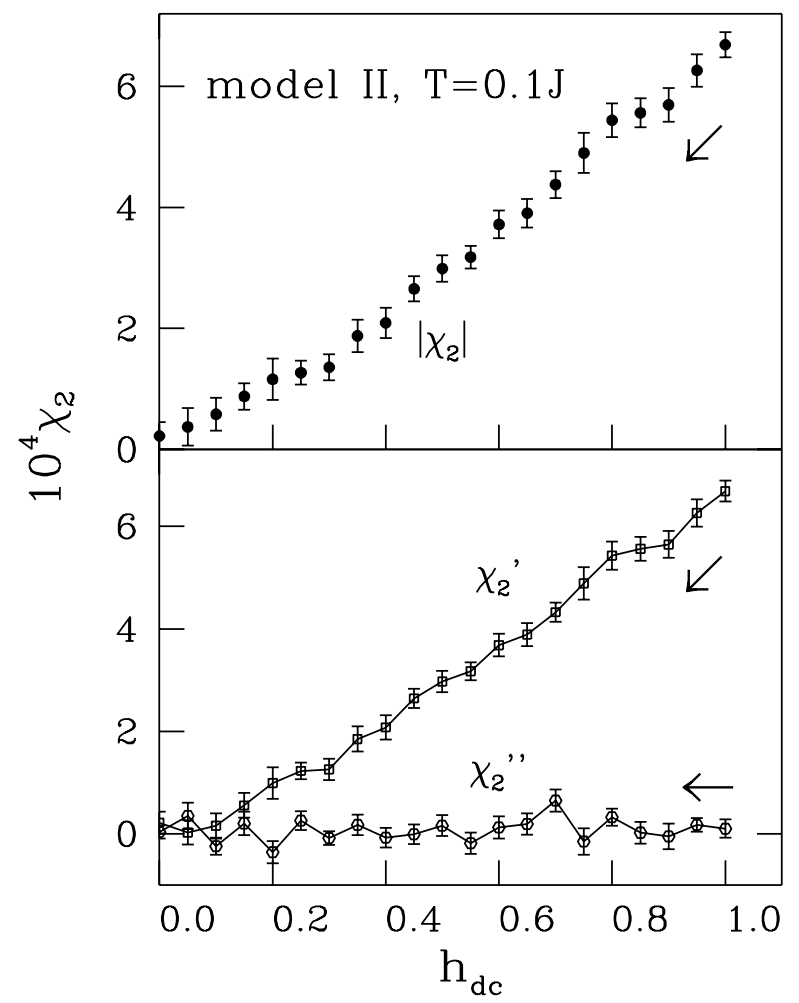

FIG. 3. The procedure to generate the second harmonics is the same as in Fig. 2 but for model II. The system is cooled in a dc field $h_{d c}=1$ from $T=1.4$ to $T=0.1$. The results are averaged over 20 samples.

The dependence of $\left|\chi_{2}\right|,\left|\chi_{2}\right|=\sqrt{\left(\chi_{2}^{\prime}\right)^{2}+\left(\chi_{2}^{\prime \prime}\right)^{2}}$, on $h_{d c}$ at $\underset{\tilde{L}}{T}=0.1 \mathrm{~J}$ is presented in Fig.1. For small values of $\tilde{\mathcal{L}}$, the oscillation of $\left|\chi_{2}\right|$ shows up. Such oscillation has been found for the two-dimensional superconductors in Ref. [19] and its nature is related to the lattice periodicity. Our new observation is that the oscillatory behavior is still present in the superconductors with 0 - and $\pi$-junctions (model I) but to less extent compared to model II. It is clear from Fig. 1 that $\left|\chi_{2}\right|$ does not decrease at large $h_{d c}$ but gets saturated. This is an artifact of the assumption that the Josephson contact energies $J_{i j}$ are field-independent. The field dependence of $J_{i j}$ should remove the saturation of $\left|\chi_{2}\right|$ at strong dc fields 19.21.

In order to study the difference between model I and model II through the $\mathrm{CE}$ we have to consider the weak field region where the PME may be observed. For model I the PME appears clearly for $h_{d c} \leq 1$ [7]. So the largest $h_{d c}$ we take is 1 . In this weak field regime there is no periodicity of $\left|\chi_{2}\right|$ versus $h_{d c}$ which may complicate the study of the CE. The chiral glass phase is found to exist below a critical value of the inductance $\tilde{\mathcal{L}}_{c}$ where $5 \leq \tilde{\mathcal{L}}_{c} \leq 7$ 16. One has to choose, therefore, an $\overline{\tilde{\mathcal{L}}}$ which is smaller than its critical value and in what follows we take $\tilde{\mathcal{L}}=4$.

In this paper we focus on the system size $L=8$, $\tilde{\mathcal{L}}=4, \omega=0.001$, and $T=0.1$. Our preliminary studies show that the qualitative results do not depend on the choise of the parameters of the system.

Fig. 2 shows the dependence of second harmonics on $h_{d c}$ in the FC regime for the superconductors described by model I. Our calculations follow exactly the experimental procedure of Heinzel et al [15. First the system is cooled in the dc field $h_{d c}=1$ from $T=0.7$ down to $T=0.1$ which is below the paramagnet-chiral glass transition temperature $T_{c} \approx$ 0.17 [16. The temperature step is chosen to be equal to 0.05 . At each temperature, the system is evolved through $2 \times 10^{4}$ Monte Carlo steps. When the lowest temperature is reached the dc field used in cooling is switched off and we apply the combined field given by Eq. (3). We monitor the second harmonics reducing the dc field from $h_{d c}=1$ to zero stepwise by an amount of $\Delta h_{d c}=0.05 .\left|\chi_{2}\right|$ reaches minimum at the compensation field $h_{\text {com }}=0.7 \pm 0.05$. At this point, similar to the experimental findings [15], the intersection of $\chi_{2}^{\prime}$ and $\chi_{2}^{\prime \prime}$ is observed. This fact indicates that at $H_{\text {com }}$ the system is really in the compensated state. Furthermore, in accord with the experiments, at the compensation point the real and imaginary parts should change their sign [15]. Our results show that $\chi_{2}^{\prime}$ changes its sign roughly at $h_{d c}=h_{c o m}$. A similar behavior is also displayed by $\chi_{2}^{\prime \prime}$ but it is harder to observe due to a smaller amplitude of $\chi_{2}^{\prime \prime}$.

Fig. 3 shows the dependence of the second harmonics on $h_{d c}$ in FC regime for model II. The calculations are carried out in the same way as for model I. A difference is that we start to cool the system from $T=1.4$ which is above the superconducting transition point $T_{s} \approx 0.9\left(T_{s}\right.$ is estimated from the maximum of the specific heat 7 for $\tilde{\mathcal{L}}=4$ and the results are not shown here). The temperature step is set equal to 0.1. Obviously, $\left|\chi_{2}\right|$ decreases with decreasing $h_{d c}$ monotonically. Thus, there is no CE because the remanent magnetization does not ap- 
pear in the cooling process. This result is again in accord with the experimental data [15].

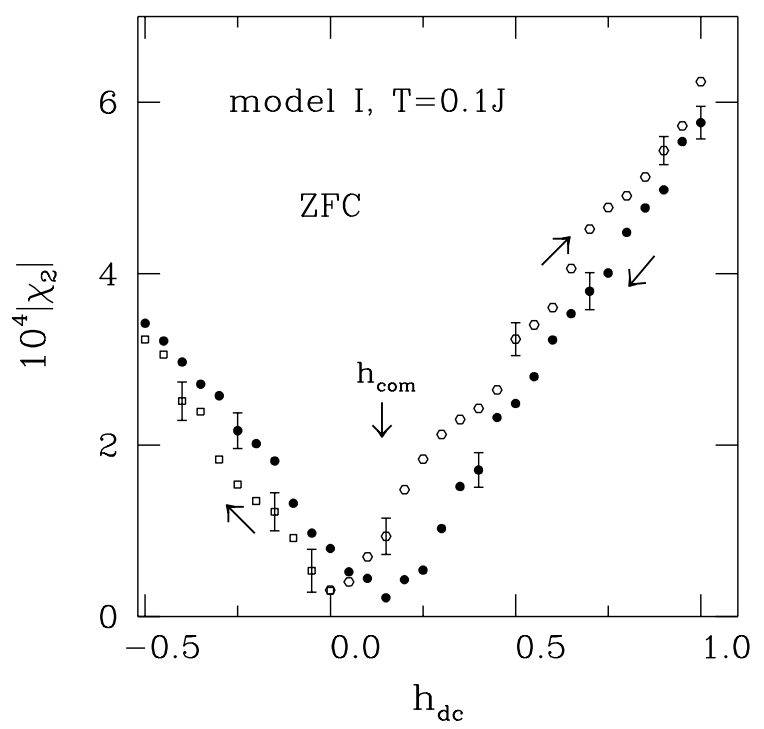

FIG. 4. The dependence of $\left|\chi_{2}\right|$ on $h_{d c}$ obtained in the ZFC regime for model I. The solid circles correspond to the case when the dc field is decreased from $h_{d c}=1$ to -0.5. The open hexagons and squares correspond to the increase of $h_{d c}$ from zero to 1 and to its decrease from zero to -0.5 , respectively. The inductance is chosen to be equal to $\tilde{\mathcal{L}}=4$. The sense of changes of the dc field is marked by the arrows. The results are averaged over 25 samples. The compensation field in the case when the field is decreased is $h_{\text {com }}=0.15 \pm 0.05$.

We now turn to the ZFC regime. The experiments [15] show that no $\mathrm{CE}$ can be expected if after the ZFC procedure one increases the dc field. However, if the field is decreased a remanent magnetization is developed and the CE appears [15]. The results of our simulations for the ceramic superconductors described by model I are shown in Fig. 4. As in the $\mathrm{FC}$ regime the system is cooled from $T=0.7$ to $T=0.1$ but without the external field. Then at $T=0.1$ we apply the field given by Eq. (3) and study three cases. In one of them $h_{d c}$ is decreased from $h_{d c}=1$ to -0.5 . The values of $\left|\chi_{2}\right|$ are represented by solid circles in Fig. 4. The CE is clearly seen at $h_{\text {com }}=0.15 \pm 0.05$. At this point the real and imaginary parts of the second harmonics also intersect (the results are not shown). It is not surprising that the $h_{\text {com }}$ in the ZFC regime appears to be smaller than in the $\mathrm{FC}$ regime. Fig. 4 shows also the dependence of $\left|\chi_{2}\right|$ on the dc field when it changes from $h_{d c}=0$ to 1 (open hexagons) and from $h_{d c}=0$ to -0.5 (open squares). Obviously, no $\mathrm{CE}$ is observed in this case. The results presented in Fig. 4 qualitatively agree with those shown in Fig.2 of Ref. [15].

In conclusion we have shown that the $\mathrm{CE}$ may be explained, at least qualitatively, by using the chiral glass picture of the ceramic superconductors. The $\mathrm{CE}$ is shown to appear in the chiral glass phase in which the PME is present but not in the samples without the PME.

We thank M. Cieplak for a critical reading of manuscript and H. Kawamura, D. Dominguez, A. Majhofer and S. Shenoy for discussions. Financial support from the Polish agency KBN (Grant number 2P03B-025-13) is acknowledged.

[1] P. Svedlindh, K. Niskanen, P. Nordblad, L. Lundgren, B. Lönnberg and T. Lundström, Physica C 162-164 (1989) 1365.

[2] W. Braunish, N. Knauf, V. Kataev, S. Neuhausen, A. Grutz, A. Kock, B. Roden, D. Khomskii, and D. Wollleben, Phys. Rev. Lett. 68, 1908 (1992); W. Braunisch, N. Knauf, G. Bauer, A. Kock, A. Becker, B. Freitag, A. Grütz, V. Kataev, S. Neuhausen, B. Roden, D. Khomskii, D. Wohlleben, J. Bock and E. Preisler, Phys. Rev. B 48 (1993) 4030.

[3] F.V. Kusmartsev, Phys. Rev. Lett. 69 (1992) 2268; J. of Superconductivity 5 (1992) 463.

[4] L. N. Bulaevskii, V. V. Kuzii, and A. A. Sobyanin, JETP Lett. 25, 290 (1977); V. B. Geshkenbein, A. I. Larkin, and A. Barone, Phys. Rev. B 36, 235 (1987); B. I. Spivak and S. A. Kivelson, Phys. Rev. B 43, 3740 (1991).

[5] M. Sigrist and T.M. Rice, J. Phys. Soc. Jpn. 61 (1992) 4283; Rev. Mod. Phys. 67 (1995) 503

[6] D. Domínguez, E.A. Jagla and C.A. Balseiro, Phys. Rev. Lett. 72 (1994) 2773.

[7] H. Kawamura and M.S. Li, Phys. Rev. B 54 (1996) 619.

[8] D. J. Thompson, M. S. M. Minhaj, L. E. Wegner, and J. T. Chen, Phys. Rev. Lett. 75, 529 (1995).

[9] P. Kostic, B. Veal, A. P. Paulikas, U. Welp, V. R. Todt, C. Gu, U. Geiser, J. M. Williams, K. D. Carlson, and R. A. Klemm, Phys. Rev. B 53, 791 (1996); 55, 14649 (1997).

[10] L. Pust, L. E. Wegner, and M. R. Koblischka, Phys. Rev. B 58, 14191 (1998).

[11] A. K. Geim, S. V. Dubonos, J. G. S. Lok, M. Henini, and J. C. Maan, Nature 396, 144 (1998).

[12] A. E. Koshelev and A. I. Larkin, Phys. Rev. B 52, 
13559 (1995); A. E. Khalil, Phys. Rev. B 55, 6625 (1997).

[13] V. V. Moshchalkov, X. G. Qui, and V. Bruyndoncz, Phys. Rev. B 55, 11793 (1997).

[14] M. Sigrist, Nature 396, 110 (1998).

[15] Ch. Heinzel, Th. Theilig, and P. Ziemann, Phys. Rev. B 48, 3445 (1993).

[16] H. Kawamura and M. S. Li, Phys. Rev. Lett. 78, 1556 (1997).

[17] H. Kawamura and M. S. Li, J. Phys. Soc. Jpn 66, 2110 (1997).

[18] L. Leylekian, M. Ocio and J. Hammann, Physica C 185-189, 2243 (1991); Physica B 194-196, 1865 (1994); M. Matsuura, M. Kawachi, K. Miyoshi, M. Hagiwara, and K. Koyama, J. Phys.Soc. Jpn 64,, 4540 (1995).

[19] T. Wolf and A. Majhofer, Phys. Rev. B 47, 5383 (1993).

[20] M. Z. Cieplak, T. R. Gawron, and M. Cieplak, Phys. Rev. B 39, 6757 (1989); M. S. Li, T. Q. Hung, and M. Cieplak, J. Phys. (France) I 6, 1 (1996)

[21] L. Ji, R. H. Sohn, G. C. Spalding, C. J. Lobb, and M. Tinkham, Phys. Rev. B 40, 10936 (1989) 